\title{
THE STUDY OF THERMAL REVERSIBILITY OF THE FREEZING-DEFROST PROCESS OF BROWNED ONION
}

\author{
Oleksander Cherevko \\ Kharkiv State University of Food Technology and Trade \\ 333, Klochkovs'ka str., Kharkiv, Ukraine, 61051 \\ rector_hduht@kharkov.com \\ Andrey Odarchenko \\ Department of commodity science, quality management and ecological safety \\ Kharkiv State University of Food Technology and Trade \\ 333, Klochkovs'ka str., Kharkiv, Ukraine, 61051 \\ 3494545@ukr.net \\ Nycolay Pogozhikh \\ Department of energetics and physics \\ Kharkiv State University of Food Technology and Trade \\ 333, Klochkovs'ka str., Kharkiv, Ukraine, 61051 \\ padnip@gmail.com \\ Dmytro Odarchenko \\ Department of commodity science, quality management and ecological safety \\ Kharkiv State University of Food Technology and Trade \\ 333, Klochkovs'ka str., Kharkiv, Ukraine, 61051 \\ laboratory119@mail.ru \\ Evgenia Sokolova \\ Department of commodity science, quality management and ecological safety \\ Kharkiv State University of Food Technology and Trade \\ 333, Klochkovs'ka str., Kharkiv, Ukraine, 61051 \\ evgenia-sokolova@ukr.net
}

\begin{abstract}
The features and dynamics of the freezing-defrost processes of the studied sample of browned onion were established and studied. At the research it was revealed, that at freezing temperature $-20{ }^{\circ} \mathrm{C}$ the value of maximal freezing speed does not change.

The process of defrost of browned onion was carried out using calorimeter with the reverse connection on temperature and PID-regulator, on the temperature curve of its freezing. It was determined, that the process of defrost of the studied sample needs more heat that is educed at freezing. The modes of stepped defrost that testify to the possibility of thermal reversibility of freezing process were experimentally established.

The direct dependence of educed and consumed heat quantity on the mode of thermal processing of studied sample was revealed. The more moisture was eliminated from the food product, the less heat must be consumed for defrost. The analogous dependence is typical also for the freezing process.

The received data can be used for determination of the rational modes of freezing and defrost of the browned onion.

Keywords: browned onion, freezing, thermal reversibility, vegetable raw material, defrost.

DOI: $10.21303 / 2504-5695.2016 .00169$

(C) Oleksander Cherevko, Andrey Odarchenko, Nycolay Pogozhikh, Dmytro Odarchenko, Evgenia Sokolova
\end{abstract}

\section{Introduction}

Freezing it is the one of most widespread and progressive method for the food products storage [1]. Using the correct methods of freezing, food products preserve most part of vitamins, their 
chemical composition is approximated to the initial raw material, they have the rather long storage life and preserve the natural color, taste and proper marketable look [2].

The main parameters that influence the quality of frozen products are temperature and speed of freezing. The speed of freezing as the main parameter characterizes the degree of effectiveness of heat exchange between cooling medium and frozen product that determines the energetic effectiveness of the process [3].

Nowadays the tendency of canned products manufacturing is at the stage of changing their consumption properties to the increase of readiness degree [4].

Technology of manufacturing of the frozen food products with the high readiness degree provides already the use of thermal processing. The one of popular methods of thermal processing is browning. It is used for softening of product. At the expense of temperature increase the elimination of some moisture, change of density and increase of porosity take place in the product.

In this connection the study of thermal reversibility of freezing-defrost process is topical. That is defrost must be carried out in such a way that one or several parameters of the raw material have the same kinetics as at freezing [5]. Based on it, the aim of the work was formulated. It is the study of conditions of thermal reversibility of freezing-defrost processes of browned onion.

\section{Materials and Methods}

As the subject of research there was used the raw material of vegetable origin, namely the onion of the German selectionists' sort (Studgarten, Rizen) Fig. 1, planted in Ukraine, in Kharkov region, that underwent browning and low-temperature freezing and defrost. The selected sort of onion is characterized with the high content of dry substances; it contains the large quantity of Vitamin $\mathrm{C}$ and is also suitable for processing and storage.

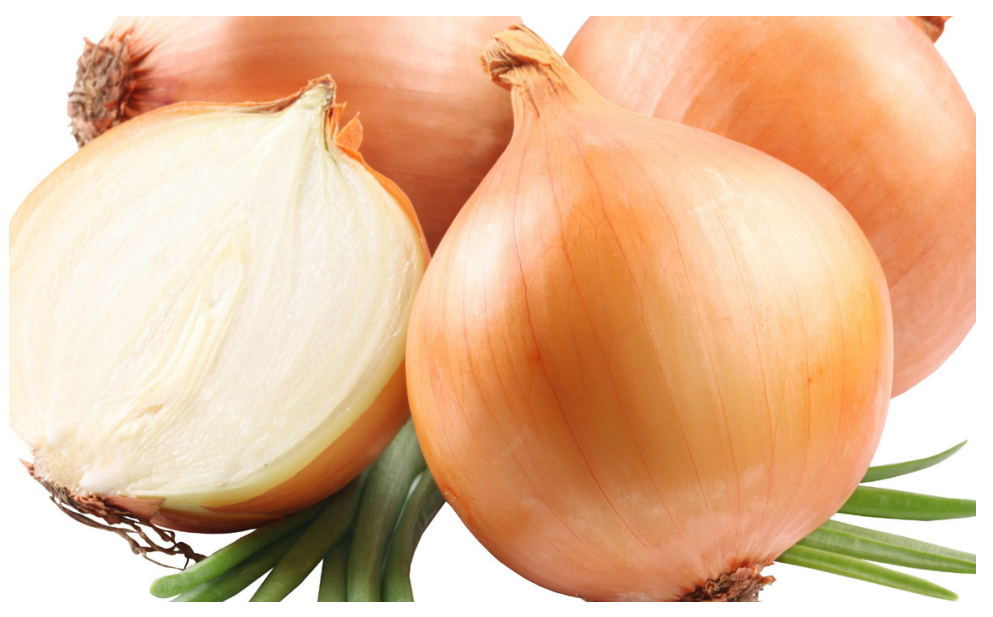

Fig. 1. Onion of German selectionists' sort (Studgarten, Rizen)

Freezing was carried out using experimental setting - low-temperature calorimeter, made in Institute of cryobiology and cryomedicine of NAS of Ukraine and patented by the scientists of Kharkov state university of food technology and trade, Fig. 2.

Calorimeter allows regulate the temperature and speed of freezing and also continuously register the temperature of samples, used at further analysis. The vapor of liquid nitrogen was used as cool-carrier that was mixed in equal proportion with air to receive the temperature of gas mixture $-20{ }^{\circ} \mathrm{C}$. The scheme of this setting is presented on the Fig. 3 .

The principle of work of the setting is following: nitrogen vapor enters in capsule with the help of heater that is placed in Dewar vessel 11. The temperature in capsule depends on the speed of nitrogen evaporation that, in its turn, is determined by the heater power 12 . It gives a possibility to widen the diapason of working temperatures to the values, close to the liquid nitrogen temperature. As the result of heater 9 work, the nitrogen vapor comes through the measuring camera 4 , where it absorbs heat, educed by the object. The differences of temperatures on input and output are fixed 
by the differential thermocouple using the continuous recorder 15 . The area under curve is a dependence of temperature difference on time; it is proportional to the heat quantity, educed by the object at attaining the capsule temperature. Such calorimeter gives a possibility to receive the exact results of researches, qualitative characteristics and wide temperature diapason [6].

The process of defrost was carried out using the low-temperature calorimeter using proportional-integral-differential (PID) regulator that allows decrease the value and duration of sample temperature derivation from the given one. The scheme of the system of automatic regulation with reverse connection is given on the Fig. 4.

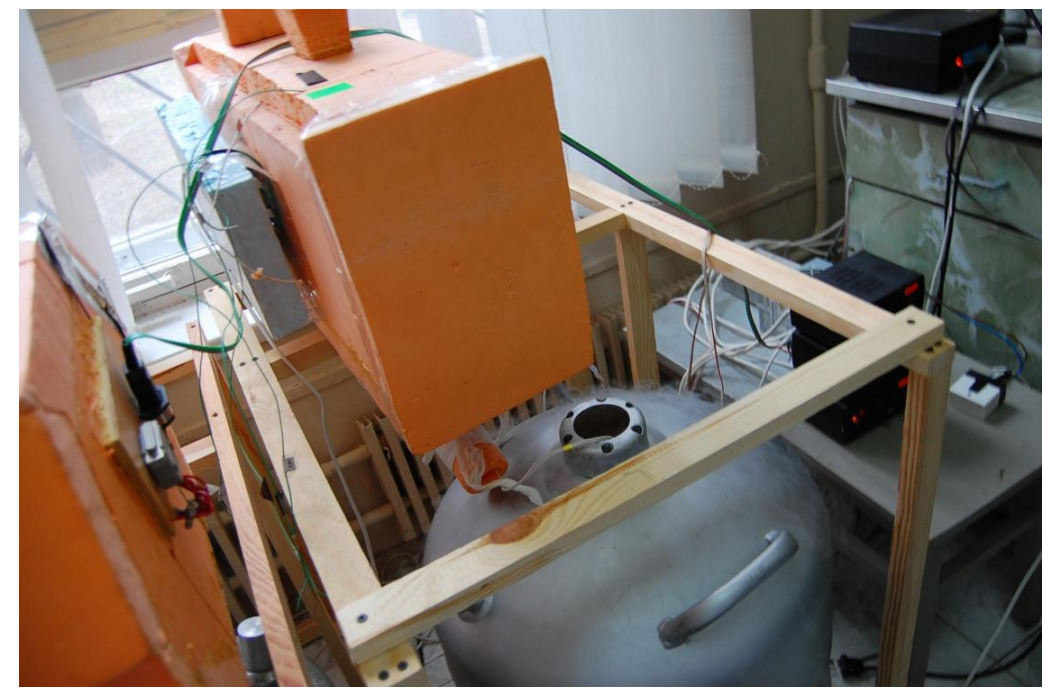

Fig. 2. Experimental setting - low-temperature calorimeter

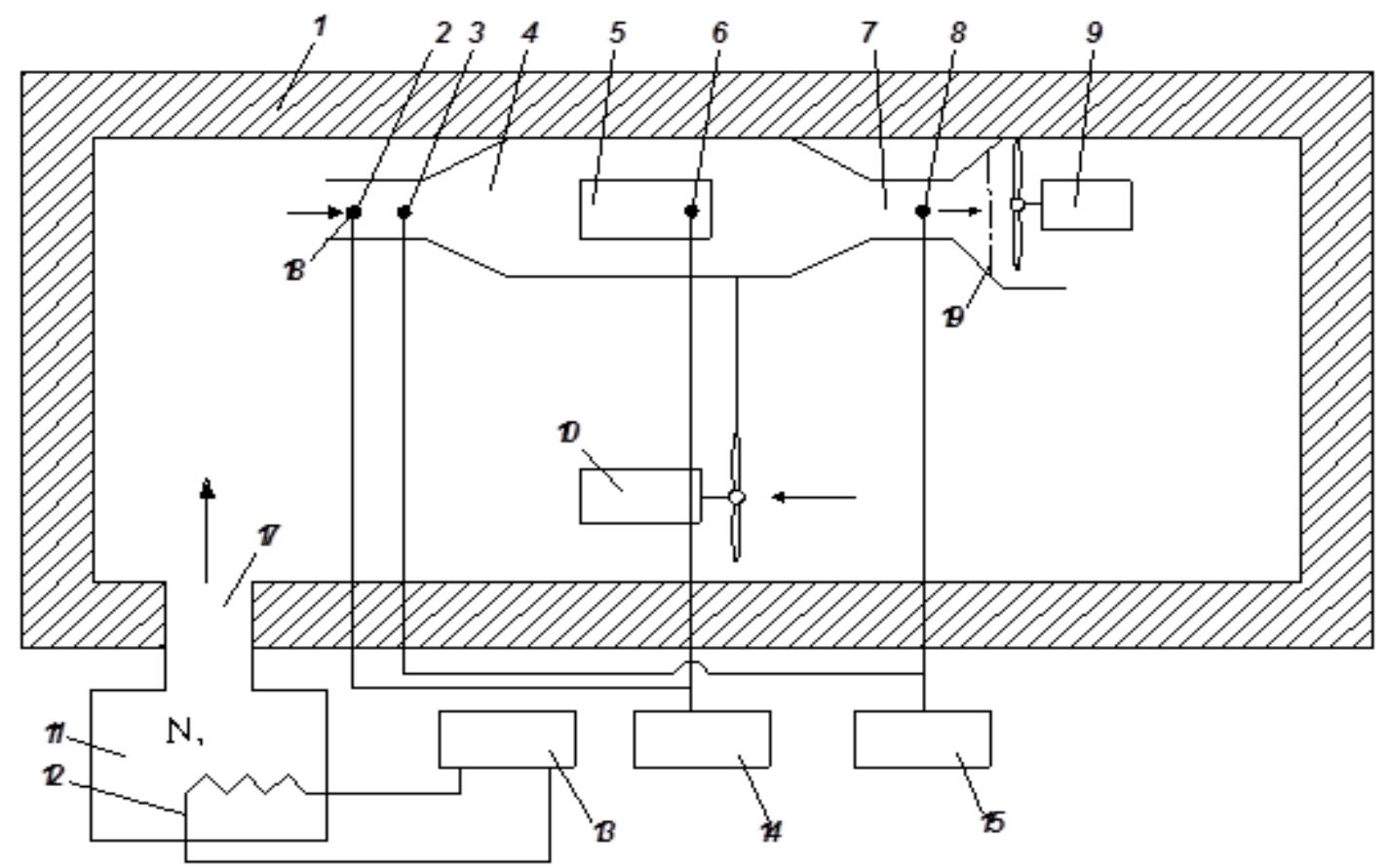

Fig. 3. Scheme of low-temperature calorimeter for freezing: 1 - capsule; 2, 7 - input and output orifice of measuring camera; 3, 8 - junctions of differential thermocouple; 4 - measuring camera; 5 -studied object; 6, 17 -junctions of thermocouples; 9, 10 -ventilators; 11 - Dewar vessel; 12 - heater; 13 - autotransformer; 14, 15 - electronic recorders; 16 - input orifice; 18 - damper 


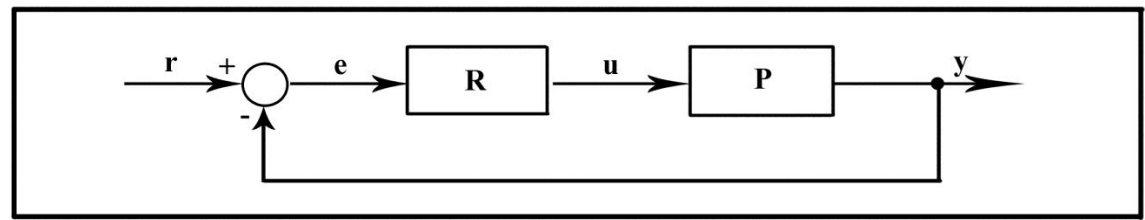

Fig. 4. PID-regulator in system with reverse connection: $\mathrm{R}$ - regulator; $\mathrm{P}$ - object of regulation; $\mathrm{r}$ - controller of influence or setting; e - controller of signal of mismatch or mistake; $\mathrm{u}$ - controller of output value of regulator; $\mathrm{y}$ - controller of regulated value

PID-regulator measures the derivation of stabilized value from the given value and generates the controlling signal that is a sum of three summands, the first of which is proportional to this derivation, second one is proportional to integral of derivation and the third one is proportional to derivative of derivation [7].

At that the proportional component is used for elimination of direct mistake in the stabilized value, observed at the present moment. The value of this component is directly proportional to the derivation of measured value from the given one [8].

As far as the equal mass second consumption of coolant was kept in the low-temperature calorimeter at freezing and defrost the heat, educed at heating and freezing of the studied samples can be compared. At that the heat quantity can be determined by the formula (1):

$$
\mathrm{Q}_{\text {heating, freezing. }}=\mathrm{M}^{\prime} \mathrm{Cp} \int_{\tau_{1}}^{\tau_{2}} \Delta \mathrm{t}(\tau) \mathrm{d} \tau \text {, }
$$

where $\mathrm{M}^{\prime}$ - mass second consumption of coolant, $\mathrm{kg} / \mathrm{s}, \mathrm{Cp}$ - thermal capacity of coolant, $\mathrm{J} / \mathrm{kg} \cdot \mathrm{K}$, $\Delta \mathrm{t}(\tau)$ - difference of temperatures of coolant on input-output of calorimeter, $\mathrm{K}$.

At $\mathrm{M}^{\prime}=$ const and $\mathrm{Cp}=$ const, integral would determine the heat quantity, consumed for heating. Determining this integral even in relative units, one can judge about the ratio of heat values, consumed on each area.

Registration was realized using chromel-copel thermocouples with junction diameter $0,2 \mathrm{~mm}$. The signal from thermocouple was registered by the digital potentiometer MAX5160, made by MAXIM-DALLAS USA, connected with PC port CelE1400/1Gb/160Gb. Statistical processing of the data was carried out using MathCad 14 software.

\section{1. Experimental procedures}

The studied sample of browned onion as crushed homogenous mass in quantity $20 \mathrm{~g}$ was frozen by immersion in calorimeter with specified medium temperature (Fig. 5).

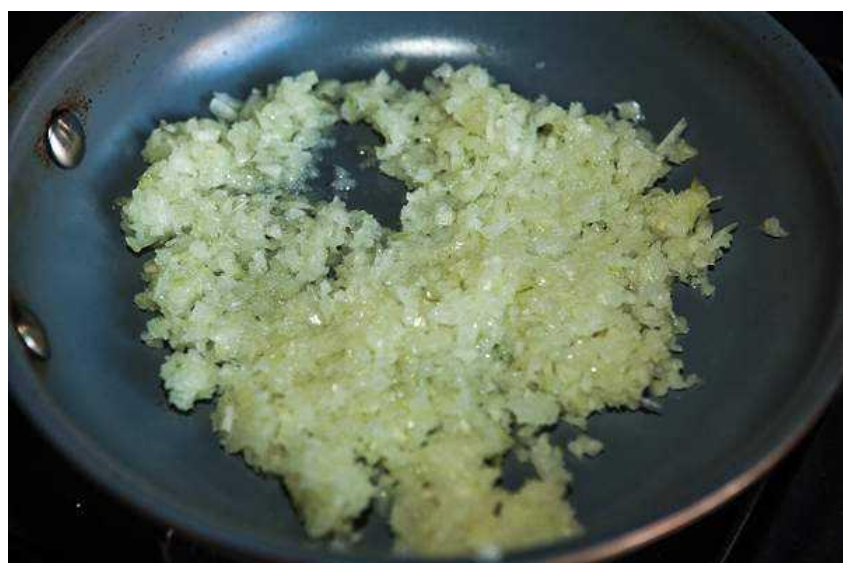

Fig. 5. Studied sample of browned onion 
Technological preparation of onion was carried out according to the following technological scheme: inspection, washing, peeling, crushing and browning. The readiness mode was determined according to organoleptic parameters. In the result the duration of preliminary technological processing was established for each browning mode: to transparency 0,6 the browning duration must be 5 min.; mode 1,0-8 min.; mode 1,2-10 min., mode 1,9-15 min. The mode of onion browning 1,0-8 was selected for the researches.

The low-temperature calorimeter freezes in the temperature diapasons from $-20{ }^{\circ} \mathrm{C}$ to $-70{ }^{\circ} \mathrm{C}$. The freezing temperature $-20^{\circ} \mathrm{C}$ was selected for experiment.

The general look of freezing thermogram (to the temperature $-20{ }^{\circ} \mathrm{C}$ ) and heating one (to the temperature $+20 \pm 2{ }^{\circ} \mathrm{C}$ ) of the studied sample is presented on the Fig. 6 .

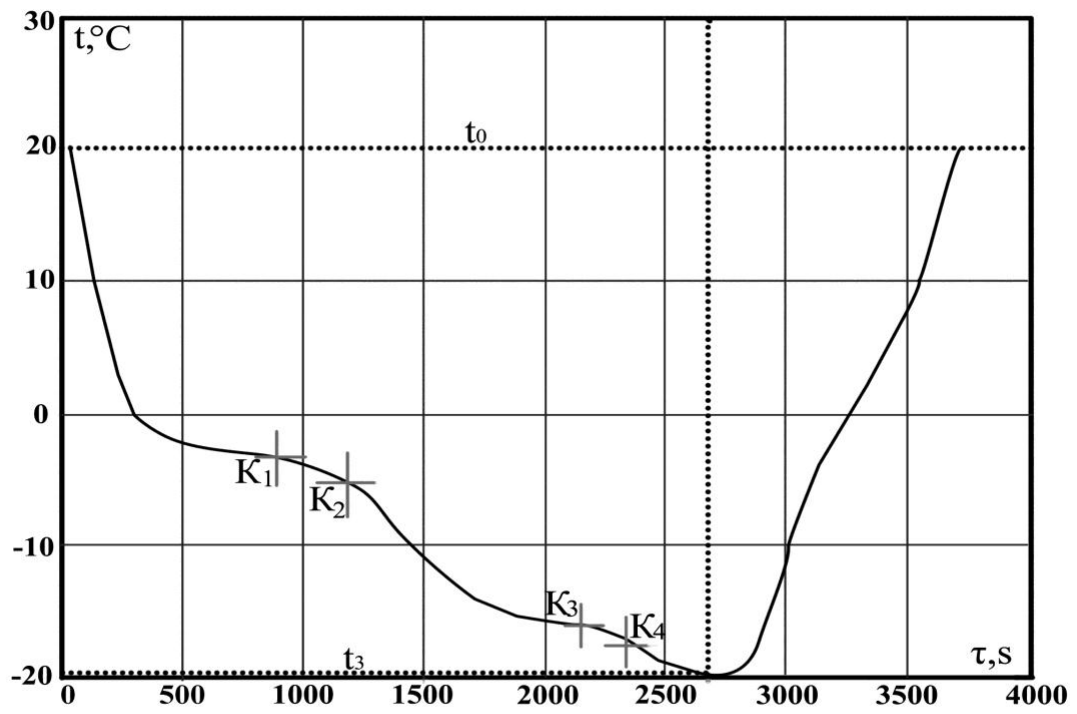

Fig. 6. The mean temperature of sample at freezing (to $\mathrm{t}-20^{\circ} \mathrm{C}$ ) and defrost (to the room temperature): $\mathrm{K}_{1}$ - cooling of sample to the beginning of ice creation; $\mathrm{K}_{2}$ - process of crystallization of the part of chilled water; $\mathrm{K}_{3}$ - cooling of sample and crystallization of non-chilled water; $\mathrm{K}_{4}-$ cooling of sample to the freezing temperature $-20^{\circ} \mathrm{C}$

The received thermogram is divided in two areas. The left part of thermogram it is the area of freezing at freezing temperature $\left(-20^{\circ} \mathrm{C}\right)$, that is a constant one, the right part - the area of defrost. Defrost temperature is equal to $+20 \pm 2{ }^{\circ} \mathrm{C}$.

It is worth noting, that the duration of freezing process is two times more than defrost. The curves have not the symmetry relative to the time scale, the divergence between them is explained by the difference between thermal conductivities of ice and water. The thermal conductivity of ice is 4 times more than the one of liquid water and coefficient of thermal conductivity of defrosted product is 2 times more than of the frozen one [9]. The internal temperature of product increases to the point of ice melting and remains constant, then increases to the general level $4-5{ }^{\circ} \mathrm{C}$.

The four typical areas can be seen on the critical points of all curves:

1. From the beginning of freezing to the point $\mathrm{K}_{1}$ - cooling of sample to the beginning of ice creation.

2. From the point $\mathrm{K}_{1}$ to the point $\mathrm{K}_{2}$ the process of crystallization of the part (chilled) of water takes place.

3. From the point $\mathrm{K}_{2}$ to the point $\mathrm{K}_{3}$ - cooling of sample and beginning of crystallization of (non-chilled) water.

4. From the point $\mathrm{K}_{4}$ the cooling of sample directly to the freezing temperature $-20{ }^{\circ} \mathrm{C}$ takes place.

The freezing speed of the studied sample is presented on the Fig. 7. 


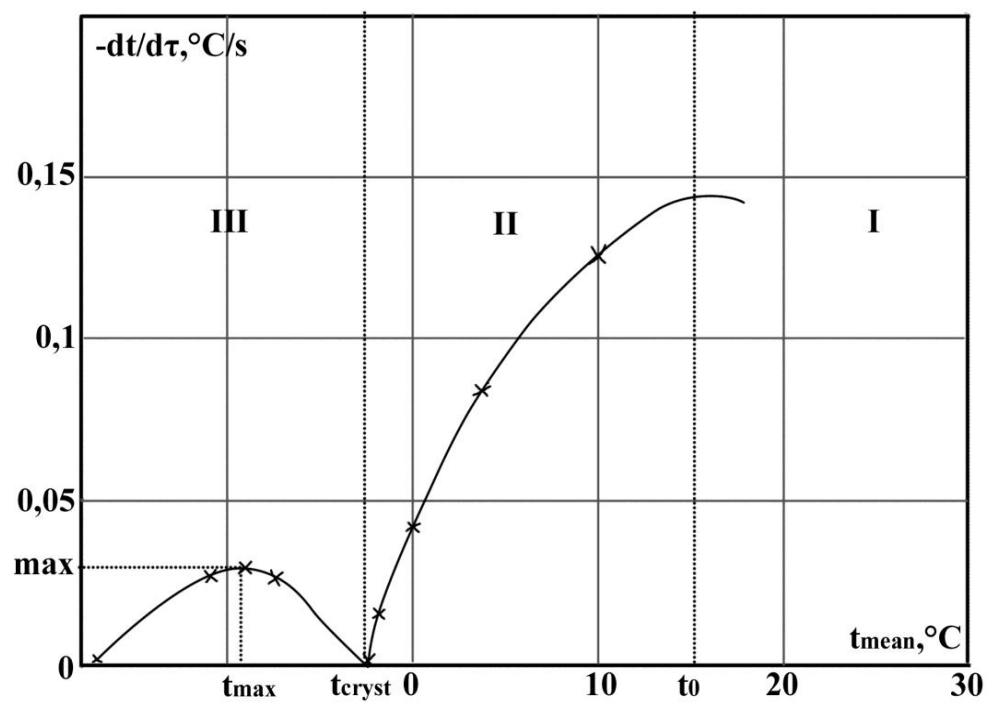

Fig. 7. Freezing speed of the studied sample of browned onion: $t_{0}$ - initial temperature of the sample; $\mathrm{t}_{\text {cryst }}$ - temperature of sample at the water crystallization; $\mathrm{t}_{\max }$ - temperature of sample at the maximal freezing speed; I - initial state of the sample; II - cooling of sample to the temperature of crystallization; III - cooling of sample to the temperature of freezing

Freezing speed depends on thermalphysic characteristics of the browned onion, speed of movement of the cooling medium and temperature. The freezing speed decreases depending on the temperature of object and at absence of the phase transitions. At crystallization (phase transitions) the heat is educed and the temperature of sample is approximated to the crystallization one. It is a result of the fact that the temperature of sample does not changeand the cooling speed approaches zero (Fig. 7).

After crystallization the thermalphysic characteristics of the studied sample change, its temperature behavior is as it is presented on the area III (Fig. 4). The speed of temperature changes varies non-linearly depending on the temperature of sample itself, it has maximum (max) in the area of low temperatures. Maximal freezing speed is characterized with the thermal conductivity of sample that is the more temperature speed, the more thermal conductivity of sample [10].

Maximal freezing speed at temperature $-20^{\circ} \mathrm{C}$ was $0,021 \pm 0,002{ }^{\circ} \mathrm{C} / \mathrm{s}$, crystallization points were observed at $-5,4{ }^{\circ} \mathrm{C}$.

The typical curve in coordinates $\Delta \mathrm{t}-\tau$ for the processes of freezing-defrost of the studied sample of browned onion is presented on the Fig. 8 .

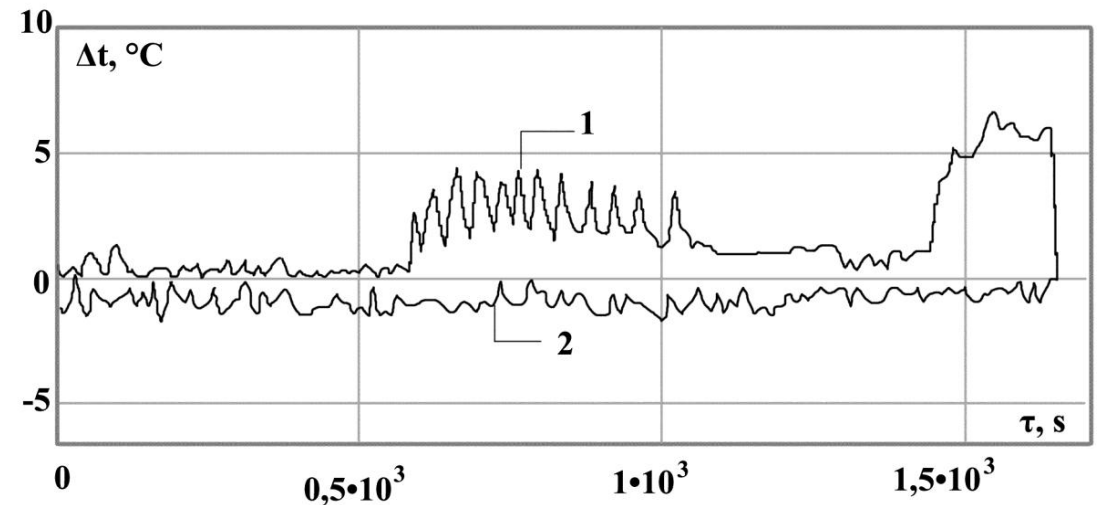

Fig. 8. The difference of temperature on input-output of calorimeter for the process of freezing-defrost of the studied sample of browned onion 1 - defrost process, 2 - freezing process 
The presented thermogram is divided in 2 areas: the graph, situated above $0{ }^{\circ} \mathrm{C}$, corresponds to the defrost process and the one below $0{ }^{\circ} \mathrm{C}$ - to freezing. The given areas of curves differed; that means that more heat was consumed for defrost of the studied sample of onion then it was educed at freezing of it.

According to the received thermogram (Fig. 8), the calculation of average weighted areas, limited by the graphs of freezing-defrost, was carried out. The relative error in calculations of the heat quantity was $\pm 10 \%$. Based on these calculations, the relative quantity of heat, educed at the onion freezing $460 \pm 46 \mathrm{~K} \cdot \mathrm{s}$, and the quantity of heat, consumed at defrost $490 \pm 49 \mathrm{~K} \cdot \mathrm{s}$.

At the same time it is necessary to note, that the use of PID-regulator allowed decrease the given power at approaching of the temperature of studied sample to the specified temperature of defrost and also reduce the consumption of cool-carrier.

The graph, situated above $0{ }^{\circ} \mathrm{C}$ of the thermogram (Fig. 8) was conventionally divided in 3 areas and the heat quantity, consumed at each stage of defrost, was calculated. It gave a possibility to determine the modes of stepped heating of studied sample that would allow repeat the character of heating temperature that practically coincides with the temperature of sample at freezing (Fig. 9), that is realize the thermal reversibility of the freezing-defrost process.

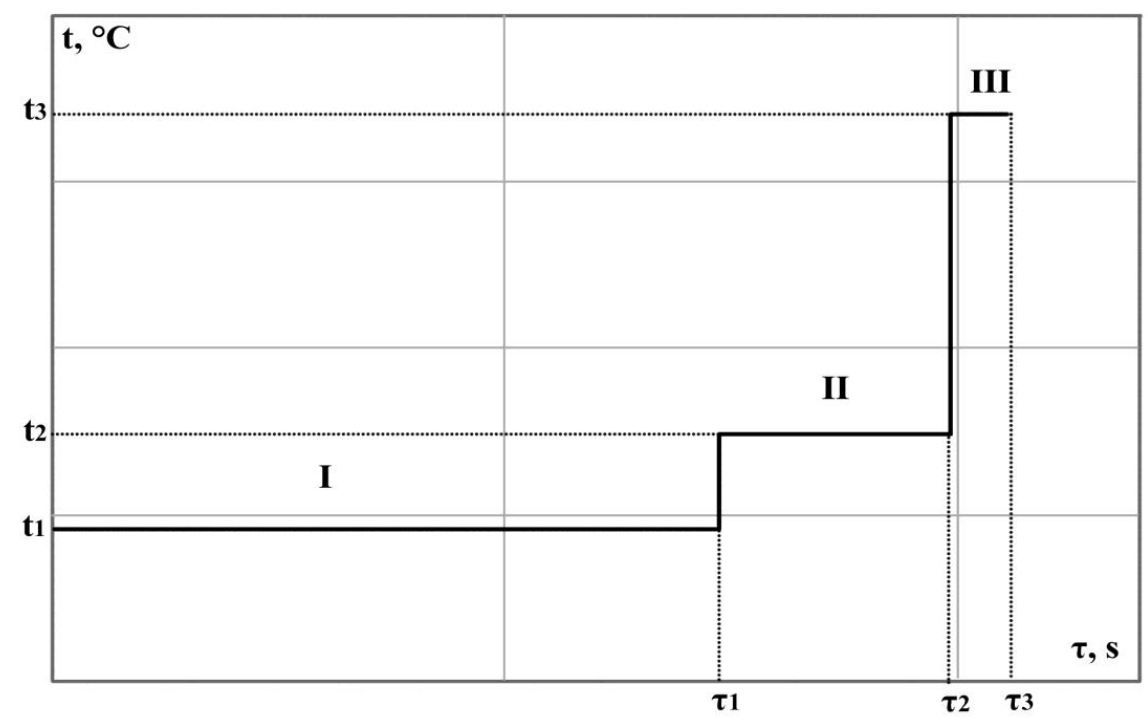

Fig. 9. Graph of stepped defrost of the browned onion

It is worth noting that: the most duration is observed at the I stage of defrost, when the temperature on input of calorimeter is $-3 \ldots-10{ }^{\circ} \mathrm{C}$, the less - at III stage, at $\mathrm{t}_{\text {input }}=15 \ldots 25^{\circ} \mathrm{C}$.

The modes of stepped defrost of browned onion are presented in the Table 1.

Table 1

The modes of stepped defrost of browned onion

\begin{tabular}{lcccc}
\hline Studied sample & & I stage & II stage & III stage \\
\hline \multirow{2}{*}{ Browned onion } & $\mathrm{t},{ }^{\circ} \mathrm{C}$ & -17 & -11 & 10 \\
& $\tau, \mathrm{s}$ & 710 & 670 & 270
\end{tabular}

This stepped mode allows maximally approach the process of freezing-defrost of the sample to the thermal reversibility: avoid hysteresis in kinetics. 


\section{Results}

In the result of freezing at the temperature $-20{ }^{\circ} \mathrm{C}$ the value of maximal freezing speed does not changed that testifies to the fact that rate of cooling is not limited by thermophysic properties of the sample. In the studied sample of browned onion the crystallization temperature of chilled water is displaced in the direct dependence on the freezing temperature. The maximal freezing speed at the temperature $-20{ }^{\circ} \mathrm{C}$ was $0,021 \pm 0,002{ }^{\circ} \mathrm{C} / \mathrm{s}$, crystallization points were observed at $-5,4{ }^{\circ} \mathrm{C}$.

The features of freezing-defrost processes of the studied onion sample were established. It was revealed, that the freezing of studied sample needs $1,5-5$ more heat that it is educed at freezing.

It is necessary to note that: the most duration of defrost is observed at the I stage, when the temperature on input of calorimeter is $-3 \ldots-10^{\circ} \mathrm{C}$, the less - at III stage, at $t_{\text {input }}=15 \ldots 25^{\circ} \mathrm{C}$. The presence of three steps testifies to the phase transitions of water and change of thermalphysic properties of the vegetable raw material near these transitions.

The direct dependence between educed and consumed heat quantity on the mode of thermal processing of studied sample was revealed. The more moisture was eliminated from the food product, the less heat must be consumed for defrost. The analogous dependence is typical also for the freezing process.

\section{Conclusions}

1. It was established, that the preliminary thermal processing, namely browning, before freezing improves organoleptic parameters that are determinant ones taking into account the functional-technological properties of the frozen vegetable half-finished product.

2. At the researches it was revealed, that at freezing temperature $-20{ }^{\circ} \mathrm{C}$ the value of maximal freezing speed does not change that testifies to the fact that the rate of cooling is not limited by thermophysic properties of the sample. In the studied sample of browned onion the crystallization temperature of chilled water is displaced in the direct dependence on the freezing temperature.

3. The process of defrost of the browned onion was carried out using calorimeter with the reverse connection on temperature and PID-regulator, on the temperature curve of its freezing. It was determined, that defrost of studied sample needs more heat that it is educed at freezing of onion. Based on calculations, the related quantity of heat, educed in the process of onion freezing was $460 \pm 46 \mathrm{~K} \cdot \mathrm{s}$, and the quantity of heat, consumed at defrost was $490 \pm 49 \mathrm{~K} \cdot \mathrm{s}$.

4. The modes of stepped defrost that testify to the possibility of realization of thermal reversibility of the process were established.

5. The received data can be used to provide the high organoleptic quality parameters of the frozen vegetable mixes and to determine the rational modes of freezing and defrost of the browned onion in their composition.

6. In further it is possible to develop and improve this direction of research for widening of assortment and formation of the high marketable-technological parameters of the frozen vegetable mixes. It is also expedient to use the system studies of behavior of the different physical-chemical, microbiological and other properties of vegetable raw material at the defined techniques and modes of technological processing.

\section{References}

[1] Reid, D. S., Neel, S. (2008). World Food Logistics. WFLO Commodity Storage Manual, 1-5.

[2] Sikora, E., Cieslik, E., Filipiak-Florkiewicz, A., Leszczynska T. (2012). Effect of hydrothermal processing on phenolic acids and flavonols contents in selected brassica vegetables. Acta Sci. Pol., Technol. Aliment, 11 (1), 45-51. 
[3] Lisiewska, Z., Gębczyński, P., Kmiecik, W. (2006). Effects of the methods of pre-treatment before freezing on the retention of chlorophylls in frozen leaf vegetables prepared for consumption. European Food Research and Technology, 226 (1-2), 25-31. doi: 10.1007/s00217-006-0504-9

[4] Pham, Q. T. (2006). Modelling heat and mass transfer in frozen foods: a review. International Journal of Refrigeration, 29 (6), 876-888. doi: 10.1016/j.ijrefrig.2006.01.013

[5] Huan, Z., He, S., Ma, Y. (2003). Numerical simulation and analysis for quick-frozen food processing. Journal of Food Engineering, 60 (3), 267-273. doi: 10.1016/s0260-8774(03)00047-5

[6] Patent № 13953 Ukraine, MPK A/23L 1/00. Device for determining the amount of free and bound water at temperatures close to the temperature of liquid nitrogen. № 200511091; 23.11.2005; 17.04.2006. Bul. 4, 6 .

[7] Barbosa-Cánovas, G. V., Altunakar, B, Mejía-Lorío, D. J. (2005). Freezing of Fruits and Vegetables: An Agribusiness Alternative for Rural and Semi-Rural Areas. Rome, 65

[8] Delgado, A. E., Sun, D.-W. (2001). Heat and mass transfer models for predicting freezing processes - a review. Journal of Food Engineering, 47 (3), 157-174. doi: 10.1016/s0260-8774(00)00112-6

[9] Giannakourou, M. C., Taoukis, P. S. (2003). Kinetic modelling of vitamin C loss in frozen green vegetables under variable storage conditions. Food Chemistry, 83 (1), 33-41. doi: 10.1016/s03088146(03)00033-5

[10] Ninfali, P., Bacchiocca, M. (2003). Polyphenols and Antioxidant Capacity of Vegetables under Fresh and Frozen Conditions. Journal of Agricultural and Food Chemistry, 51 (8), 2222-2226. doi: 10.1021/jf020936m 